\title{
Nüchterne Perspektiven auf die Reformfähigkeit des Bundesstaates
}

\author{
Merten, Detlef (Hrsg.): Die Zukunft des Föderalismus in Deutschland und Europa, Duncker \& \\ Humblot, Berlin 2007, 251 Seiten, € 94,-.
}

Der vorliegende Band dokumentiert die Verhandlungen der 73. Staatswissenschaftlichen Fortbildungstagung, die an der Deutschen Hochschule für Verwaltungswissenschaften in Speyer im März 2005 stattfand. So global der Titel anmutet, so spezifisch erscheint der Zeitpunkt der Veranstaltung: Trafen sich die teilnehmenden Staatswissenschaftler und -praktiker doch in einer Phase, in der das Scheitern der „Kommission zur Modernisierung der bundesstaatlichen Ordnung" (KoMbO) unmittelbar nachwirkte, zugleich aber eine „stille Kompromissfindung“ zwischen Bundes- und Landesexekutiven greifbar nahe schien. Wie dynamisch sich der weitere Prozess der Föderalismusreform gestalten würde - zunächst das vermeintliche Ende nach der Ankündigung der vorgezogenen Bundestagswahl und dann die unerwartet zügige Umsetzung unter der Großen Koalition -, war in der damaligen Situation nicht abzusehen. Von daher könnte man den Eindruck gewinnen, dass es sich bei dem Buch um einen der zahlreichen Sammelbände handelt, der bereits bei Erscheinen veraltet war und somit keine gesonderte Aufmerksamkeit verdient. Dies ist jedoch nicht der Fall: Nicht nur vermitteln die Beiträge interessante Einschätzungen zu den Arbeiten der $\mathrm{KoMbO}$, sondern sie verweisen auch auf Strukturprobleme, die nach der ,großen“ Föderalismusreform von 2006 fortbestehen.

Im ersten Beitrag stellt Klaus Stern die bundesstaatliche Ordnung auf den Prüfstand. Sein „Rezept“ lautet: „Rückkehr zum kompetitiven Föderalismus mit transparenten politischen Strukturen, der den Vätern und Müttern des Grundgesetzes 1949 vorschwebte“ (S. 20). Angesichts der Analysen von Gerhard Lehmbruch oder Hans Boldt, die die Prägekraft unitarischer Leitideen im Parlamentarischen Rat herausgearbeitet haben, wirkt diese Zielvorstellung stark idealtypisierend. Sterns nachfolgende Argumentation ist jedoch weit differenzierter. Während er der Rahmengesetzgebung ihre Daseinsberechtigung abspricht und an der Neufassung des Art. 84 Abs. 1 GG jegliche „Verfassungsästhetik“ vermisst, steht er einer vollständigen Abschaffung der Gemeinschaftsaufgaben durchaus skeptisch gegenüber. Und die allfällige Neuordnung des Länderfinanzausgleichs hält er für eine „Quadratur des Kreises“, „der zu entrinnen wohl nur durch die Neugliederung des Bundesgebietes gelingt“ (S. 30).

Anschließend arbeitet Horst Risse, Leiter des KoMbO-Sekretariats, heraus, wie die inhaltlichen Positionen einzelner Akteure zu erklären sind und welche Lösungen sich folglich innerhalb der KoMbO abzeichneten. Demnach wäre der Kommission keine vollständige Abschaffung der Rahmengesetzgebung gelungen, weil sich Bund und Länder bei den umweltrelevanten Materien und beim Hochschulrecht nicht auf eine Neuverteilung der entsprechenden Kompetenzen einigen konnten. Eine Abschaffung der Gemeinschaftsaufgaben, über die anfangs breiter Konsens bestand, scheiterte an „Unsicherheiten über die Kompensationsleistungen des Bundes“ (S. 62). Und obwohl in der Kommission über die unzureichende Wahrnehmung deutscher Interessen auf EU-Ebene Einigkeit herrschte, verhinderte die „feste Rollenverteilung“ zwischen Bund und Ländern eine Änderung der europapolitischen Kompetenzordnung (Art. 23 GG).

Georg-Bernd Oschatz befasst sich mit der Kultur- und Bildungspolitik im deutschen Bundesstaat. Seine Untersuchung der in die Kritik geratenen Kultusministerkonferenz 
(KMK) führt zu einem interessanten Befund: Verantwortlich für die Funktionsprobleme der KMK sei weniger die Vielfalt der mitwirkenden Gremien als das Abhandenkommen des tradierten Grundkonsenses, was Bildung, Ausbildung und Kultur ist (S. 80). Dies wiederum mündet in ein Plädoyer für eine „gewisse Zurückdrängung des Staates“ (S. 83) aus dem Bildungs- und Kulturbereich, wobei aber die neuen Grenzen zwischen staatlicher Verantwortung und (zivil)gesellschaftlicher Autonomie offen bleiben.

Mit dem Aufsatz von Ewald Wiederin zur Föderalismusreform in Österreich wird die Diskussion auf erhellende Weise um eine vergleichende Perspektive erweitert. Wie der Autor anhand des „Österreich-Konvents“ aufzeigt, der zeitgleich zur KoMbO eine neue Bundesverfassung ausarbeiten sollte, gab es gravierende Unterschiede: Während in Deutschland das Leitbild der Entflechtung dominierte, war in Österreich ein Trend zu einer stärkeren Verantwortungsteilung zwischen Bund und Ländern erkennbar. Zudem sei der Konvent nicht an einem Konflikt um einzelne Kompetenzfelder gescheitert, sondern an der Weigerung der Bundesseite, den Ländern mehr Mitwirkungsrechte auf gesamtstaatlicher Ebene zuzugestehen (S. 113).

Die drei folgenden Beiträge erscheinen eher konventionell, insofern als die darin vertretenen Positionen großenteils aus der bisherigen Reformdebatte bekannt sind. Hans-Jürgen Papier unterzieht die wichtigsten Reformvorschläge der KoMbO einer kritischen Würdigung. Da hierdurch keine "grundlegende Änderung der derzeitigen, wenig befriedigenden Situation“ zu erreichen sei, verweist er auf zwei „grundsätzlichere Überlegungen“ (S. 132): das Senatsmodell als Alternative zum Bundesrat sowie eine Neugliederung des Bundesgebiets. Unter welchen funktionalen und politischen Rahmenbedingungen diese Ideen handlungsleitend werden könnten, bleibt aber offen. Auch Edzard Schmidt-Jortzig sieht in einer klaren Zuordnung der Verantwortlichkeiten auf Bundes- oder Landesebene das „Hauptziel einer Föderalismusreform“. Diesbezüglich habe die KoMbO „durchaus respektable Ergebnisse“ (S. 148) zustande gebracht. Ob sich jedoch durch die Rückverlagerung eher kleinteiliger Gesetzgebungszuständigkeiten an die Länder „eine größere Rechtsvielfalt im Bundesgebiet" (S. 149) ergibt, wie der Autor optimistisch prognostiziert, scheint nach den jüngsten Erfahrungen eher zweifelhaft. Demgegenüber liegt das Hauptaugenmerk von Ferdinand Kirchhof auf der bundesstaatlichen Finanzverfassung. Aus seiner Sicht führt die in der KoMbO dominierende Zielvorstellung der Aufgabenentflechtung „letztlich zur Idee des fiscal federalism, der Aufgaben, Ausgaben und Einnahmen in einem Sachbereich stets nur einer Gebietskörperschaft zuweisen will“ (S. 169). Rein theoretisch wäre dies am besten durch „die primäre Zuweisung von Steuerquellen und die sekundäre Erstattung von Durchschnittskosten bei der Finanzierung übertragener Aufgaben“ (S. 172) zu erreichen. Angesichts (europa-)rechtlicher und funktionaler Restriktionen bestünden die wichtigsten Reformoptionen in einer Neuordnung der Ertragshoheit, einer Verminderung des Finanzausgleichs, der Rückführung der Bundesfinanzhilfen sowie der Abschaffung der Gemeinschaftsaufgaben. So sehr diese Argumentation theoretisch überzeugt, so wenig konkrete Ansatzpunkte bietet sie für politisch kompromissfähige Handlungsoptionen.

Daraufhin plädiert Christoph Grimm, Landtagspräsident von Rheinland-Pfalz, für eine Stärkung der Landesparlamente im Mehrebenensystem. Dies sei nicht nur verfassungspolitisch geboten, sondern zunehmend auch verfassungsrechtlich notwendig, da die fortschreitende europäische Integration den Mindestbestand an autonomen Länderkompetenzen bedrohe und damit das Bundesstaats- wie das Demokratieprinzip des Grundgesetzes beeinträchtige. Einen Ausweg sieht er in einem Reformvorschlag der Landtagspräsidentinnen 
und -präsidenten von 1991, nach dem die Mitglieder des Bundesrates bei der Übertragung von Gesetzgebungszuständigkeiten „an Beschlüsse der Landesparlamente gebunden“ werden sollten (S. 192). In sachlich unmittelbarem Anschluss diskutiert Peter M. Huber die „Europatauglichkeit des Art. 23 GG“ und kommt dabei zu einem gleichermaßen kritischen wie pointiert formulierten Gesamturteil. Leider erreicht Hubers Argumentation hier nicht die gewohnte Präzision und systematische Kohärenz, da offenbar nur ein Wortprotokoll des mündlichen Beitrags ohne ersichtliche redaktionelle Bearbeitung abgedruckt wurde.

Eine nachträgliche Analyse der Grundgesetzänderungen von 2006 beschließt den Tagungsband. Darin zieht Wito Schwanengel eine vernichtende Bilanz: Die Entflechtungswirkung sei unzureichend, die Abweichungsgesetzgebung paradox, der Abbau von Zustimmungserfordernissen zirkulär und der Systembruch der Gemeinschaftsaufgaben nur partiell beseitigt - alles in allem eine „mutlos[e], in wichtigen Punkten inkonsequent[e] und konzeptionell unausgereift[e]“ Reform (S. 249). An Positivem bleibt nur ein ironischer Vergleich mit dem Wahrzeichen Barcelonas: der Sagrada Família, an der seit über 100 Jahren gebaut wird und von der der Volksmund sagt, dass ihre Fertigstellung auch das Ende der Stadt bedeuten würde. Von daher „dürfte dem deutschen Bundesstaat noch ein langes Leben beschieden sein“" (S. 249).

Insgesamt reiht sich der Band nahtlos in die „blaue Reihe“ der Speyerer Schriften ein, in denen prominente Autoren zum aktuellen Zustand der bundesstaatlichen Ordnung Stellung beziehen. Selbst wenn man nicht mit allen vorgetragenen Positionen übereinstimmen mag, liefert das Buch auch mit zeitlichem Abstand einen wichtigen Beitrag zu einem historisch wie materiell fundierten Verständnis des deutschen Föderalismus.

Florian Grotz

\section{Instruktive Wortmeldung zur Debatte über die Föderalismusreform II}

Junkernheinrich, Markus, Henrik Scheller und Matthias Woisin (Hrsg.): Zwischen Reformidee und Funktionsanspruch. Konzeptionen und Positionen zur deutschen Finanzverfassung (Forum Öffentliche Finanzen 11), Analytica Verlagsgesellschaft, Berlin 2007, 347 Seiten, € 49,90.

Im Sommer 2006 gelang der Großen Koalition die Einigung auf eine Reform des deutschen Föderalismus, die Rot-Grün ein gutes Jahr zuvor wegen des Widerstands aus Hessen noch missglückt war. Die Föderalismusreform I wurde dem Publikum als großer Wurf präsentiert. Die Inkonsistenz, Zufälligkeit und Zaghaftigkeit des erzielten Ergebnisses steht zu dieser Selbsteinschätzung allerdings in deutlichem Widerspruch. Schließlich hatte die Reform nur gelingen können, weil der heikelste Punkt ausgeklammert worden war: die Umstrukturierung der Finanzverfassung. Diese derzeit in der Beratung befindliche Föderalismusreform II setzt an den eigentlichen und grundlegenden Problemen des deutschen Bundesstaates an. Dazu haben Markus Junkernheinrich, Hendrik Scheller und Matthias Woisin einen Sammelband vorgelegt. Die Mehrheit der 21 Artikel ist durch Finanzwissenschaftler, Juristen und Praktiker aus den Finanzverwaltungen von Bund und Ländern verfasst worden; Politikwissenschaftler sind kaum vertreten. 